\title{
Distributed Fiber Optical Sensing Inside the Foundation Slab of a High-Rise Building
}

\author{
Clemens Horch, Jan Hupfer, Dr. Frank Schäfer \\ Fraunhofer Institute for High-Speed Dynamics, Ernst-Mach-Institut, Eckerstr. 4, 79104 Freiburg, \\ Germany \\ clemens.horch@emi.fraunhofer.de
}

\begin{abstract}
In this paper, the results of distributed strain measurements inside the foundation slab of a high-rise building are presented. Optical fibers that have been embedded directly into the concrete by attaching them to the reinforcement are used as sensors. The measurements were obtained using a measurement system that is based on Rayleigh scattering and provides very high spatial resolution.

Prior works have shown that embedding of fiber optical sensors into concrete is possible. This paper adds an example for their application in an actual high-rise building, while most other publications focus on laboratory structures or bridges. This opens up new applications in the construction industry. Structural health monitoring and safety applications could benefit from this new technology.
\end{abstract}

Besides organizational issues, the main challenge can be identified as proper compensation of thermal induced expansion and the processing and interpretation of the resulting high-resolution data.

Key words: distributed fiber optical sensing, strain sensing, temperature compensation, high-rise buildings

\section{Distributed fiber optical strain sensing}

Over the course of the last 25 years, several different systems for distributed fiber optical sensing appeared on the market. They rely on different physical effects that describe the spectrum of backscattered light in an optical fiber. These systems use the so-called Raman, Brillouin or the Rayleigh scattering, some also a combination of those. Henault et al. [1] and Delepine-Lesoille et al. [2] give an overview on different technologies and applications. Bao and Chen [3] provide an in-depth overview of the technology available and the theoretical background of the underlying physical principles.

We chose a system based on Rayleigh scattering that uses the so-called "Optical Backscattered Reflectometry" (OBR). OBR systems are commercially available since 2006 by Luna Inc. In this project, we used a device called "ODiSI-B". The system goes back to the developments described by Froggatt et al. [4], [5]. Samiec [6] describes the system used for this work in more depth and gives an example of its application with a sensor embedded into concrete.

The configuration of the sensor system provides a spatial resolution of $5 \mathrm{~mm}$, a temporal resolution of $50 \mathrm{~Hz}$ and a maximum sensing length of $20 \mathrm{~m}$. These parameters are different from many other systems that often have much lower spatial and temporal resolutions but much higher sensor lengths. The spatial resolution allows us to obtain a very detailed view of the strain distribution. Since the width of the observed structure is $32 \mathrm{~m}$, the maximum of $20 \mathrm{~m}$ is sufficient, if two sensors are used.

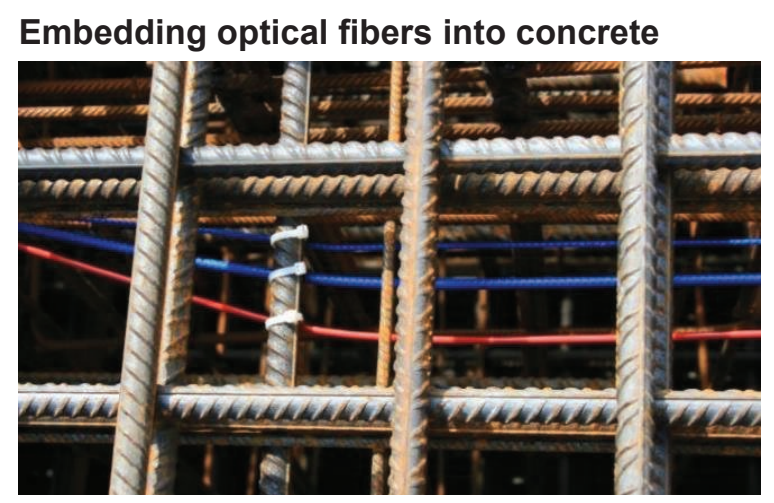

Figure 1: Three different sensor cables attached to the upper reinforcement layer (red: temperature, blue: strain)

For the evaluation of the application of distributed fiber optical strain sensing 12 sensor cables of $20 \mathrm{~m}$ each have been deployed to the foundation slab of a $140 \mathrm{~m}$ high-rise building 
during construction. The sensors cover the complete width of the building and were installed on the top and the bottom layer of the reinforcement cage (see Figure 3). The cables have been attached to the steel reinforcement using cable ties. Figure 1 shows a detail shot of the installed sensor cables. One end of each fiber is accessible from an installations room in the basement of the building. The other end is terminated reflection free and packed in a robust case because it resides in the concrete as well. Figure 3 shows the fiber termination as used in this project.

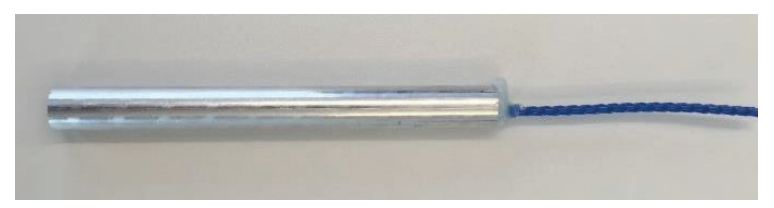

Figure 3: Robust fiber termination for embedding into concrete

The method for embedding the optical fibers into the building structure is crucial for the application of distributed fiber optical sensing. The fiber has to be well protected from the harsh environment at the construction site and especially during concrete pouring. Robust sensor cables are commercially available from Brugg Cables, Switzerland. Iten [7] describes how these cables have been developed for geotechnical applications.

In the course of this work, we compared two different strain-sensing cables from Brugg Cables with respect to their robustness and aspect of handling on a construction site. The cables have a diameter of $3.2 \mathrm{~mm}$ and $7.2 \mathrm{~mm}$. Both have a similar design, but the thicker cable has an additional steel armoring. As expected, the handling of the thinner cable is easier because it is more flexible and its weight per meter is only $14 \%$ of the thicker variant. The results show that both cables deliver identical results and both were able to withstand the harsh conditions.

\section{System setup}

All embedded fibers leave the foundation slab at a single point that is now accessible from a technical room in the basement of the building. The ends of these fibers are equipped with LC connectors. Since the ODiSI-B has only one measurement channel, the fibers are all connected to a fiber optical switch (FOS) that makes it possible to switch between the different sensors without changing the connections. It is advisable to reduce all necessary re-plugging of fiber connectors to a minimum in the dusty environment of a

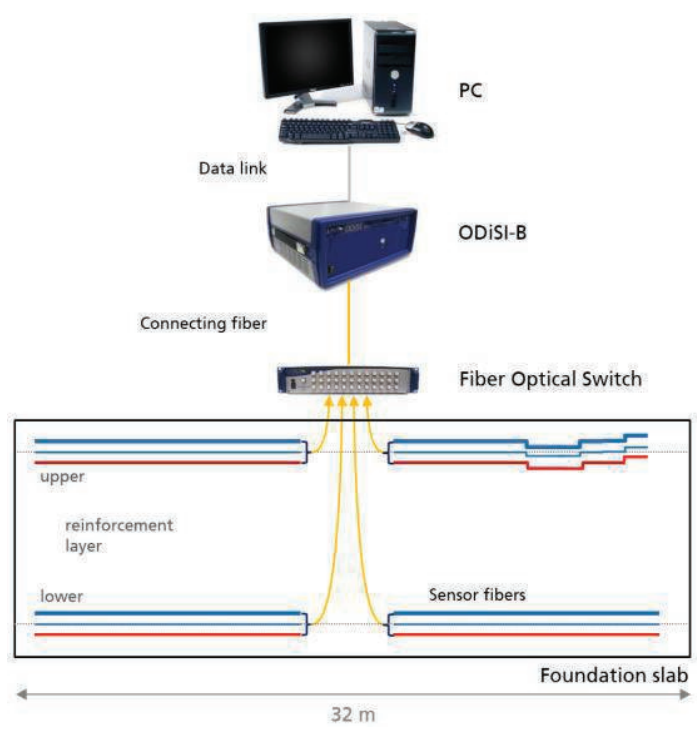

Figure 2: Location of the sensor cables in the foundation slab and experimental setup.

construction site. The FOS is then connected to the ODiSI-B device and via a data cable to the measurement PC. Figure 3 shows the complete system setup and the location of the sensor cables in the foundation slab.

Because the whole system is too expensive for a permanent installation at a construction site, we performed several measurement campaigns at intervals of several weeks. Since the deformation of the foundation slab is a slow process, it is still sufficient to perform quasistationary measurements.

Both the fibers in the upper and the lower layer do not reach completely to the right end of the slab. The reason for this is the limited measurement range of the ODiSI-B device. Especially at the lower layer, over 3 meters of the sensor cannot be used for measuring purposes because the cable has to be lowered down to the bottom of the slab.

In the upper layer, it was necessary to run the cables vertically at three points. This was due to steps in the upper surface of the foundation slab. The gray dotted vertical lines in the corresponding graph (Figure 5) mark these vertical segments.

\section{Project timeline}

Table 1 shows the complete timeline of measurements and the corresponding number of stories the building had. Until now, it has been possible to perform three measurements.

The first step after the sensor installation was a baseline measurement. At this date, we acquired a tare measurement that acts as 
baseline for all future measurements. Since it is only possible to perform relative measurements, this is a necessary step. Besides that, an important result of the baseline measurement is that nearly all installed fiber cables were still fully functional after the concrete pouring.

Table 1: Project timeline

\begin{tabular}{lll} 
Week & Stories & Event \\
\hline $\mathbf{0}$ & & Concrete pouring \\
$\mathbf{1 8}$ & 5 & Baseline measurement \\
$\mathbf{2 6}$ & 12 & First measurement \\
$\mathbf{3 4}$ & 18 & Second measurement
\end{tabular}

Eight weeks later the first real measurement was performed and six weeks after that, the second measurement. At both times, the changes in temperature in strain have been measured once.

\section{Compensation of thermal effects}

Since the expected strain values are only in the range of several $10 \mu \mathrm{m} / \mathrm{m}$, it is essential to compensate the thermal expansion of the concrete. This is also the main reason for deploying temperature-sensing fibers next to the strain cables.

Figure 4 shows the results of the temperature measurements in the upper layer as an example. Especially in the right half of the graph, strong noise can be observed. According to the datasheet of the ODiSI-B, the expected noise for temperature measurements is in the range of $\pm 0.8 \mathrm{~K}$. The measured signal has a standard deviation of $\pm 2.0 \mathrm{~K}$ in the spatial dimension across the length of the sensor.
If these strong fluctuations corresponded to actual temperature differences, the strain curve would have to show a similar behavior. Consequently, we can only assume that the reason for this lies in either the measurement system or the sensor cables. Additionally, it is not possible to use this date for compensation of the thermal expansion in dependence of the position.

On the other hand, the temperature values oscillate around a mean value that is constant across the whole foundation slab. The two horizontal lines in Figure 4 show these mean values. We used these means for a global compensation of the thermal expansion. For this purpose, a constant strain offset is calculated from the mean temperature and subtracted from the measured strain values. It is a reasonable assumption that the temperature across the width of the structure is constant since during the construction phase there is no heating or ventilation in place that could affect the temperature of the upper side of the foundation slab. At the lower level, the temperature should be constant anyway.

As thermal expansion coefficient, we assumed $10 \cdot 10^{-6} \mathrm{~K}^{-1}$, which is a common literature value. The exact value of this coefficient is another factor that can introduce additional uncertainties.

\section{Discussion}

Figure 5 shows the results for a strain sensor at each the upper and the lower reinforcement layers. The graphs show the combination of the sensing cables left and right of the point where they leave the slab. This point corresponds to position 0 in the diagram.

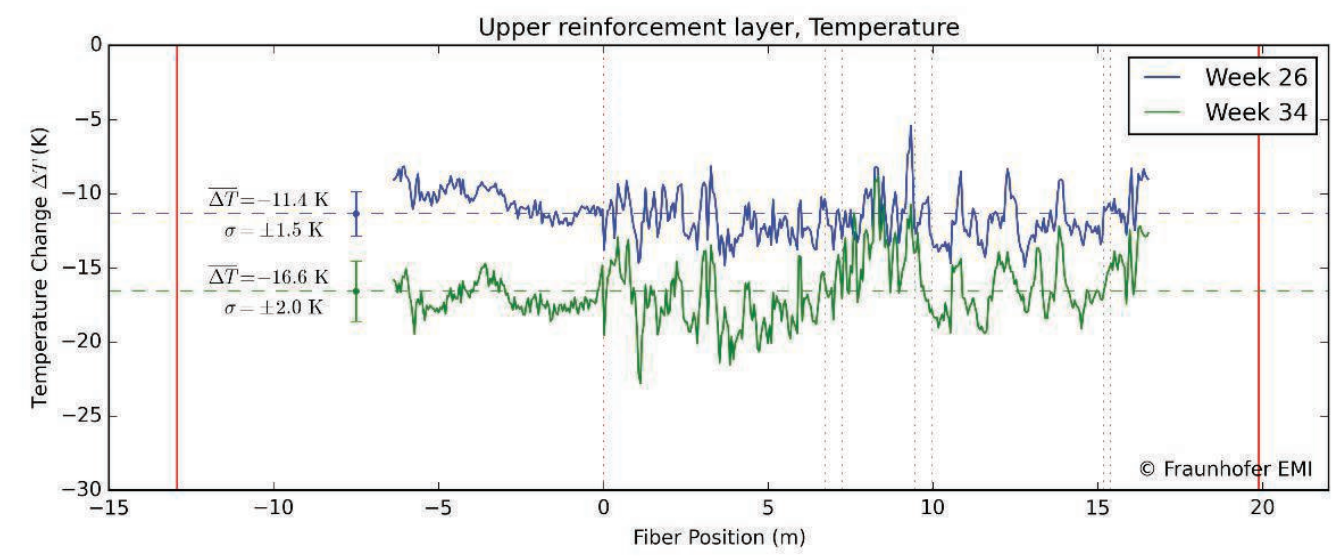

Figure 4: Results of temperature measurements at the upper reinforcement layer. The horizontal lines show the mean of the temperature curves. The solid red lines denote the dimensions of the foundation slab. Position 0 is the position where the fibers leave the slab. The dotted grey lines mark vertical sections of the sensor cable. 
Both between the baseline and the first measurement as well as between the first and the second measurement the absolute value of strain seems to increase. This is natural since the load on the foundation increases with the construction progress.

Furthermore it can be observed, that the vertical sections in the upper layers have an influence on the measured strain beyond that vertical part of the fiber. It remains to be seen whether this is really the case in the structure or an effect induced by imperfections of the sensor cable.

It is interesting that both in the upper and in the lower layer, the measured strain is negative. From the behavior of a standard cantilever, it is known that the strain is negative on the top and positive on the bottom. The reason why this is different here is still under investigation und remains unclear until now.

Another interesting fact is that the shape of the strain graphs is almost identical between the first and the second measurement but not between the baseline measurement and the first measurement. This means that certain tensions have developed between weeks 18 and 26 and have remained unchanged between weeks 26 and 34.

It has to be noted that it is not possible to make final statements about the deformation of the foundation slab based on the measurements described. Especially the temperature effects need further investigation. However, the questions after a possible influence of the sensor cables cannot be answered finally.

\section{Conclusion}

The central part of this work is to demonstrate the use of distributed fiber optical sensing inside a real building as opposed to a laboratory setup. Many challenges arise from that. They mainly concern the robustness of the system and the sensors but also organizational matters.

For the interpretation of the data gathered, further investigation is required. This is currently in progress, as is the whole project. Further measurements will be performed, accompanied by laboratory tests that will help to understand the data in more depth.
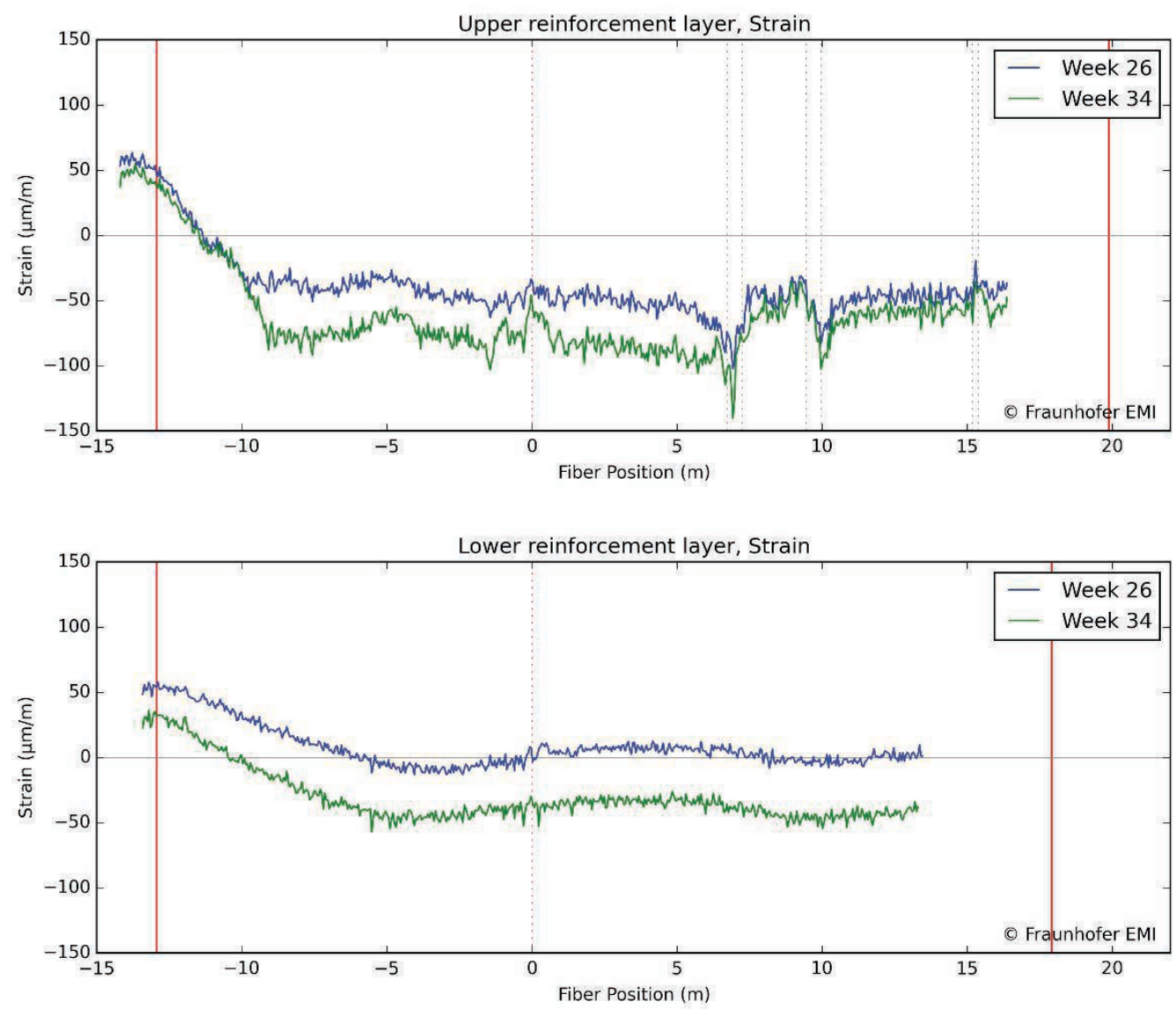

Figure 5: Results of strain measurements at the upper and the lower reinforcement layer after compensation of the thermal expansion. 
Nevertheless, distributed strain sensing has the potential to provide insightful data that otherwise would not be available. Despite its complexity, it is still much less effort to achieve this grade of resolution and accuracy than with conventional technology like resistive strain gauges.

With regard to possible applications, we can think of two main areas. Firstly, this kind of measurement technology can be interesting for constructional engineers. It is common to do calculations and simulations of structural loads, but it is difficult to validate these theoretical results in the real world. Here, this technology could fill a gap. Secondly, the fields of structural health monitoring and building safety could benefit from this technology. While it may not be justifiable from an economic point of view to equip buildings with optical sensing by default, it might be an option to do so in special areas like critical infrastructure and security and safety-critical buildings.

This work is part of the research project AURIS (Autonomes Risiko- und Informationssystem zur Strukturanalyse und Überwachung sicherheitsrelevanter Bauwerke) [8] and is funded by BMBF (German Federal Ministry of Education and Research) under Grant No. 13N11742.

\section{References}

[1] Jean-Marie Henault et al., "Truly Distributed Optical Fiber Sensors for Structural Health Monitoring: From the Telecommunication Optical Fiber Drawling Tower to Water Leakage Detection in Dikes and Concrete Structure Strain
Monitoring," Advances in Civil Engineering, 2010.

[2] Sylvie Delepine-Lesoille, Erick Merliot, and Yves Gautier, "Optical fiber strain sensors for use in civil engineering: State-of-the-art, industrial applications and outlook," vol. 272, pp. 123-140, 2008.

[3] Xiaoyi Bao and Liang Chen, "Recent Progress in Distributed Fiber Optic Sensors," Sensors, vol. 12, pp. 8601-8639, 2012.

[4] Mark Froggatt and Jason Moore, "High-spatialresolution distributed strain measurement in optical fiber with Rayleigh scatter," Applied Optics, vol. 37, no. 10, pp. 1735-1740, 1998.

[5] Mark Froggatt, Brian Soller, Dawn Gifford, and Matthew Wolfe, "Correlation and Keying of Rayleigh Scatter for Loss and Temperature Sensing in Parallel Optical Networks," in Optical Fiber Communication Conference, Los Angeles, USA, 2004

[6] Dirk Samiec, "Distributed fibre-optic temperature and strain measurement with extremely high spatial resolution," Photonic International, pp. 10-13, 2012.

[7] Michael Iten, Novel applications of distributed fiber-optic sensing in geotechnical engineering (PhD thesis). Zürich: ETH, 2011.

[8] AURIS: Autonomes Risiko- und Informationssystem zur Strukturanalyse und Überwachung sicherheitsrelevanter Bauwerke. [Online]. http://www.auris-innovation.org 